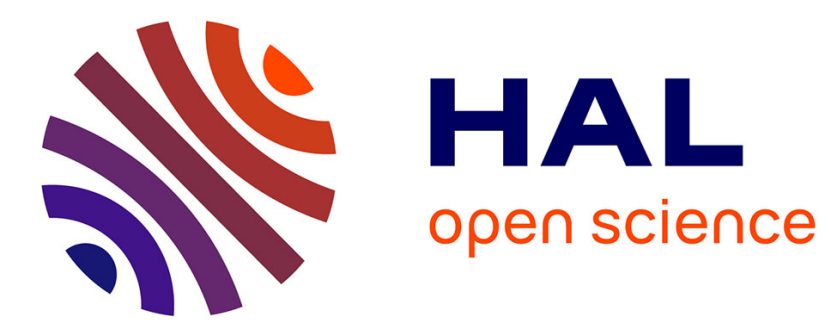

\title{
Non-neutrality in forest communities: Evolutionary and ecological determinants of tree species abundance distributions
}

\author{
Y. Kubota, B. Kusumoto, T. Shiono, W. Ulrich, F. Jabot
}

\section{- To cite this version:}

Y. Kubota, B. Kusumoto, T. Shiono, W. Ulrich, F. Jabot. Non-neutrality in forest communities: Evolutionary and ecological determinants of tree species abundance distributions. Oikos, 2016, 125 (2), pp.237-244. 10.1111/oik.02232 . hal-01743226

\author{
HAL Id: hal-01743226 \\ https://hal.science/hal-01743226
}

Submitted on 26 Mar 2018

HAL is a multi-disciplinary open access archive for the deposit and dissemination of scientific research documents, whether they are published or not. The documents may come from teaching and research institutions in France or abroad, or from public or private research centers.
L'archive ouverte pluridisciplinaire HAL, est destinée au dépôt et à la diffusion de documents scientifiques de niveau recherche, publiés ou non, émanant des établissements d'enseignement et de recherche français ou étrangers, des laboratoires publics ou privés. 
Non-neutrality in forest communities: evolutionary and ecological determinants of tree species abundance distributions

Yasuhiro Kubota $^{1 *}$, Buntarou Kusumoto ${ }^{1}$, Takayuki Shiono ${ }^{1}$, Werner Ulrich ${ }^{2}$ and Franck Jabot $^{3}$

${ }^{1}$ Faculty of Science, University of the Ryukyus, Nishihara, Okinawa, Japan

${ }^{2}$ Chair of Ecology and Biogeography, Nicolaus Copernicus University, Toruń, Poland

${ }^{3}$ Irstea, UR LISC, Campus des Cézeaux 9, Avenue Blaise Pascal - CS 2008563178

Aubière France

* Corresponding author: Yasuhiro Kubota

E-mail: kubota.yasuhiro@gmail.com

Running head: Non-neutrality in tree species abundance 


\section{Abstract}

The neutral theory of biodiversity and biogeography provides a promising framework that can be used to integrate stochastic and ecological processes operating in ecological communities. Based on the mechanistic non-neutral model that incorporates density-dependent mortality, we evaluated the deviation from a neutral pattern in tree species abundance distributions and explored the signatures of historical and ecological processes that have shaped forest biomes. We compiled a dataset documenting species abundance distributions in 1,168 plots encompassing 16,973 tree species across tropical, temperate, and boreal forests. We tested whether deviations from neutrality of species abundance distributions vary with climatic and historical conditions, and whether these patterns differ among regions. Non-neutrality in species abundance distributions was ubiquitous in tropical, temperate, and boreal forests, and regional differences in patterns of non-neutrality were significant between biomes. Species abundance evenness/unevenness caused by negative density-dependent or abiotic filtering effects had no clear macro-scale climatic drivers, although temperature was non-linearly correlated with species abundance unevenness on a global scale. These findings were not significantly biased by heterogeneity of plot data (the differences of plot area, measurement size, species richness, and the number of individuals sampled). Therefore, our results suggest that environmental filtering is not universally increasing from warm tropical to cold boreal forests, but might affect differently tree species assembly between and within biomes. Ecological processes generating particularly dominant species in local communities might be idiosyncratic or region-specific and may be associated with geography and climate. This study illustrates that mechanistic non-neutral model enables the analysis of the interplay of historical and ecological 
processes that influence community assemblies and the dynamics of biodiversity. 


\section{Introduction}

Unravelling the mechanisms that structure ecological communities has long been a central aim in ecology (Hutchinson 1959). The analysis of biodiversity gradients associated with physical environments has demonstrated the importance of species packing through local ecological processes such as habitat selection and energy availability (Currie et al. 2004). However, understanding anomalous patterns of species richness under ecologically comparable environments, so called "diversity anomalies", remains elusive and pose a significant challenge to ecologists (Jiménez and Ricklefs 2014). Geographical differences in non-neutral processes such as niche diversification, evolutionary radiation, selective extinction, and dispersal are believed to ultimately generate regional diversity anomalies among local communities (Qian and Ricklefs 2000, Harrison and Cornell 2008, Ricklefs and Renner 2012). Therefore, a key task in understanding patterns of diversity is to create a unified framework related to the origin and maintenance of biodiversity that includes local-scale ecological processes and regional-scale biogeographical, historical, and evolutionary processes (Wiens and Donoghue 2004).

In this context, Hubbell's (2001) unified neutral theory of biodiversity and biogeography provides a concrete quantitative framework for integrating local- and regional-scale processes that generate diversity gradients and anomalies (Ricklefs 2006). This theory assumes ecological equivalence of all individuals and thereby is formulated as a process-based statistical model (sampling theory) consisting of four demographic processes: individual birth, death, dispersal, and speciation (Volkov et al. 2003, Etienne 2005, Etienne and Alonso 2005). Latimer et al. (2005) used this neutral model to quantify the importance of speciation and dispersal in the highly diverse and endemic 
fynbos flora of southern Africa. Jabot and Chave (2009) further developed this model using phylogenetic information to rigorously estimate regional diversity and immigration rates. Their analysis stressed the importance of continental-scale dispersal events in shaping local diversity patterns of neotropical forests.

Ecologists have not always welcomed this radical simplification of the natural world (McGill 2010); thus, this theory is sometimes criticized as being process-free (Clark et al. 2008). Nonetheless, the concept of a neutral model also means that the model can be seen as a mechanistic null model (Gotelli and McGill 2006, Alonso et al. 2006). Additionally, the parsimony of the neutral model makes neutral theory an ideal basis from which to infer the influences of complex ecological and evolutionary processes (Alonso et al. 2006). These types of non-neutral models function as heuristic tools that can be used to evaluate the roles of niche and adaptive radiation in non-random macroecological patterns. Indeed, recent advances in the studies in neutral-based ecology investigate the impact of density dependence or environmental filtering on species abundance distributions (Volkov et al. 2005, Jabot et al. 2008, Jabot and Chave 2011, Rosindell et al. 2012), effects of speciation and long-distance dispersal on species-area relationships (Rosindell and Cornell 2009), key innovation effects on phylogenetic tree topologies (Davies et al. 2011), and community assembly as it is related to historical processes (Rosindell and Phillimore 2011).

Here, we focus on the degree of non-neutrality of tree species abundance distributions in local communities across the globe. Our aim is to test whether consistent trends exist in the way communities assemble along environmental gradients and in different biomes. Previous pioneering studies suggested that taxon origination and historical migration may influence regional divergences of forest biomes, such as 
the area of tropical biomes that persisted throughout geological times (Gentry 1988, Fine and Ree 2006). Another example can be seen in the temperate and boreal biomes that originated from particular lineages that adapted to global cooling and dispersed out of tropical Asia (as a macroevolutionary source) to the Holarctic regions during the Miocene (Latham and Ricklefs 1993, Donoghue 2008). Moreover, many local-scale studies have shown that the variability of diversity within and between biomes is operated by species sorting caused by abiotic stress and biotic pressure (e.g. Harms et al. 2000, Gilbert and Lechowicz 2004). Therefore, we can hypothesize that tree species diversity in local communities was shaped by regional combinations of in situ diversification and dispersal, and that the pattern of species abundance was further influenced by niche partitioning linked to phylogenetic niche conservatism and habitat conditions (Ricklefs and Renner 2012). Global patterns of tree communities provide an opportunity to disentangle the hierarchy of regional and local influences on anomalies and latitudinal gradients of biodiversity (ter Steege and Zagt 2002).

In this study, we assess the degree of deviation from a neutral pattern and the direction of deviation in tree species abundance distributions in relation to environmental conditions and geography. Specifically, we test (1) whether deviations from neutrality differs among regions that are characterized by different environmental conditions, and (2) how deviations from neutrality of species abundance distribution vary with climatic conditions including Quaternary climate changes. Finally, we determine how macro-scale environmental factors are associated with the evenness/unevenness of tree species abundance and discuss the geographical forces related to evolutionary and ecological determinants shaping forest communities, based on a conceptual framework unifying regional and local community perspectives. 


\section{Materials and methods}

\section{Species abundance data}

We searched the ISI Web of Science (Thomson-Reuters, New York, NY, USA) to find scientific literature using the following string: 'relative abundance' OR 'species abundance' OR ‘community’ OR ‘assemblage' OR ‘composition' OR ‘diversity or distribution'. We retrieved 92,678 publications and filtered them such that each study should: (1) be quantitative, counting tree individuals within a particular plot; and (2) contain tables of tree species composition. We also collected web-based information on forest plots. To complement the data for some regions, we compiled information from publications in non-indexed local journals using Google Scholar (http://scholar.google.com/). Note that we excluded studies that investigated only dominant species and/or that summarized rare species as "other species." From each study, we extracted plot area, minimum measured diameter at breast height (DBH), and site description, such as geographical location (latitude-longitude). The final dataset comprised 1,168 plots (mean area $=16,715 \pm 53,199 \mathrm{~m}^{2}$ ) with different minimum DBH threshold (mean DBH $=5.52 \pm 5.82 \mathrm{~cm}$ ) from 251 individual sources (Supplementary material Appendix 1 provides a list of the studies included; Appendix 2 provides detailed plot information). Appendix 3 also shows the patterns of plot area, minimum measured DBH, species richness, and the number of individuals sampled in forest plots. Species taxonomy was standardized using The Plant List (http://www.theplantlist.org/). The dataset comprised data from 3,572,064 individual trees of 16,973 species. From these data, species abundance distributions were drawn for each forest plot (Appendix 
4).

\section{Environmental data}

The 1,168 plots were classified into seven geographical regions: three in the Holarctic (Nearctic, Eastern Palearctic, and Western Palearctic) and four outside of the Holarctic (South America, Africa, Indo-Pacific, and Australia). The plots that lie outside of the Holarctic were mostly tropical (between $23^{\circ} 27^{\prime} \mathrm{N}$ and $23^{\circ} 27^{\prime} \mathrm{S}$ ). For each plot, we compiled five environmental variables (Supplementary material Appendix 2). Mean annual air temperature $\left({ }^{\circ} \mathrm{C}\right)$, precipitation $(\mathrm{mm})$, and elevation $(\mathrm{m})$ were extracted from WorldClim (http://www.worldclim.org) for each grid cell that contained a plot at a resolution of $0.5^{\circ}$ latitude by $0.5^{\circ}$ longitude. Mean air temperature $\left({ }^{\circ} \mathrm{C}\right)$ and mean annual precipitation $(\mathrm{mm})$ in the last glacial maximum were also obtained from the same database. Historical climate stability was calculated as the differences between the present day mean annual temperatures $\left({ }^{\circ} \mathrm{C}\right)$ and annual total precipitation $(\mathrm{mm})$ and those of the last glacial maximum, representing the degree of Quaternary climate changes.

\section{Statistical analysis}

Extreme care must be taken when interpreting the appearance of neutrality or non-neutrality in species abundance distribution. Finding the degree to which real communities are approximately non-neutral is essential. Therefore, we evaluated the effect size of non-neutrality to avoid dichotomous arguments of neutrality versus non-neutrality (e.g. null hypothesis tests of neutrality). 


\section{Neutral and non-neutral models}

Hubbell's neutral models assume that a local community is connected to a larger metacommunity, and claims that the species abundance distribution is shaped through the interplay of birth/death processes, dispersal, and speciation. Etienne (2005) developed an explicit sampling formula for Hubbell's neutral model with dispersal limitation. The neutral model consists of $\theta$ (fundamental biodiversity number) and $m$ (species immigration probability from a metacommunity). Note that $\theta$ is defined as two times the product of the speciation rate and the metacommunity size (Hubbell 2001), thus being closely related to the diversity of the regional species pool. Based on this framework of statistical inference, Jabot and Chave (2011) developed a non-neutral model that assumes that species with different population sizes have different mortality rates. Specifically, the model incorporates the process of density-dependent mortality into Hubbell's neutral model that formulates the transition probabilities of species abundances during one time step. The model describes the probability, $P_{j i}$, that species $i$ gains one individual while species $j$ loses one individual (with $i \neq j$ ), and is given by $P_{j i}=\frac{\left(N_{j}^{(t)}\right)^{1-\delta}}{\sum_{k=1}^{S}\left(N_{i}^{(t)}\right)^{1-\delta}} \times\left[(1-m) \frac{N_{i}^{(t)}}{J-1}+m \chi_{i}^{(t)}\right]$, where $N_{i}^{(t)}$ is the abundance of species $i$ at time $t, J$ is the local community size, and $\chi_{i}^{(t)}$ is the regional relative abundance of species $i$ at time $t . \delta$ is a new parameter representing density-dependent mortality, i.e. the degree of deviation from a neutral pattern in the species abundance distribution. When $\delta=0$, the observed species abundance distribution matches the expectation from Hubbell's neutral model. When $\delta$ is negative, individuals of dominant species face increased mortality rates compared with individuals of rare species, i.e. negative density dependence (Fig. 1a). When $\delta$ is 
positive, individuals of rare species face increased mortality rates compared with those

of dominant species (Fig. 1b). Positive density dependent mortality results in abundance unevenness, suggesting that an environmental filtering effect exists along a gradient of species fitness in relation to abiotic stresses (Jabot 2010, Jabot and Chave 2011).

\section{Parameter estimation of the non-neutral model}

In the present analysis, we fitted Jabot and Chave's (2011) non-neutral model to the species abundance distribution for each plot. We ran Approximate Bayesian Computation using 100,000 simulated species abundance distributions for each plot and retained the posterior distribution of $\delta$ values, using Parthy software (Jabot and Chave 2011). We used a uniform prior distribution for $\ln (\theta), 0 \leq \ln (\theta) \leq 25$; and $\ln (I), 0 \leq \ln (I)$ $\leq$ 10. Supplementary material Appendices 2 and 4 show the parameter estimates of $\theta, m$, and $\delta$.

Forest plot data usually include potential biases, such as differences in plot area, minimum measured diameter at breast height (DBH), and species richness sampled in the plots (Supplementary material, Appendices 2 and 3). These biases could have confounding effects on the patterns of species abundance distribution. For example, large plots are likely to cause an averaging out of environmental heterogeneity and thus cause the results to look more neutral than smaller plots (Jabot and Chave 2011). Serra et al. (2013) also indicated that a risk of incorrectly detecting the neutrality in cases such as hyperdiverse communities (e.g. tropical forests) and/or those with a very large number of individuals. Therefore, we tested for the sampling effect based on the relationships between species richness, number of individuals, and the appearance of non-neutrality $(\delta)$. 


\section{Test for patterns of non-neutrality}

To evaluate the regional differences in the parameter of the non-neutral model $(\delta)$, we tested the mean values of the parameter estimates for each region using an analysis of variance within the two biogeographical realms (Holarctic and outside the Holarctic). To evaluate the potential effect of environments on non-neutrality, we examined the correlations between $\delta$ and the environmental variables. In the multiple linear regression model, $\delta$ in each plot was set as the response variable, and temperature, precipitation, Quaternary temperature change, Quaternary precipitation change, and elevation were set as the explanatory variables. Plot area, minimum measured $\mathrm{DBH}$, the number of species, and log-scaled number of individuals were included as covariates in the model to control for their variability among plots. To remove the influence of spatial autocorrelation in the data, we also added the first eigenvector of the geographical distance matrix among the plots (Diniz-Filho and Bini 2005). To evaluate a non-linear pattern of the response variable, additionally, we examined the regression models including the quadratic and linear terms of elevation, temperature, precipitation, Quaternary temperature change, and Quaternary precipitation change.

All of the explanatory variables were standardized to have a zero mean and unit variance prior to parameter estimation. The regression analyses were applied to the global dataset as a whole and were also applied separately to the seven regions: South America, Africa, Indo-Pacific, and Australia mostly located in the tropics, and Eastern Palearctic, Nearctic, and Western Palearctic. Prior to the analysis, we tested for collinearity between the explanatory variables by calculating the determinant of the correlation matrix (D) as 0.22 , the condition index $(\mathrm{CI})$ with a maximum of 2.8 , and the 
variance inflation factor (VIF) that ranged from 1.21 to 1.94 , indicating the absence of multicollinearity in the regression model.

All analyses were performed and graphics generated using the R Environment for Statistical Computing (R Development Core Team 2012) with the 'car' (Fox and Weisberg 2014) for diagnosing multicollinearity and the 'geosphere' (Hijmans et al. 2013) for calculating geographical distance among the plots.

\section{Results}

\section{Patterns of non-neutrality in species abundance distributions}

The $\delta$ value in the non-neutrality model was not significantly correlated with species richness or the number of individuals (Fig. 2). Non-neutrality in species abundances occurred across tropical, temperate, and boreal forests (Fig. 3). The $\delta$ values substantially differed between the regions and also differed within the tropics and the Holarctic (Fig. 4 and Supplementary material Appendix 5). In South America and the Indo-Pacific region (including the tropics), the $\delta$ values tended to be positive in the majority of the plots (Fig. 4). The $\delta$ value was greatest in the Western Palearctic. The $\delta$ values were negative in $41 \%$ of all plots.

Globally and regionally, plot area, minimum measured DBH, the number of individuals, and species richness were not consistently correlated with the $\delta$ value (Table 1). Globally or in Africa and the Indo-Pacific, the $\delta$ value was related to Quaternary precipitation change, but was not strongly correlated with environmental variables (Table 1). In Africa, the $\delta$ value was positively correlated with temperature (Table 1). The quadratic terms of temperature and Quaternary precipitation change were positively correlated with the $\delta$ value in the global dataset (see Supplementary material 
Appendix 6).

\section{Discussion}

\section{Prevalence of non-neutrality in species abundance distributions}

Mechanistic process-based models that are used to explain species abundance

distributions have recently been developed within the conceptual framework of neutral ecology (Etienne 2005, Jabot et al. 2008, Jabot and Chave 2011). Nevertheless, despite the availability of an appropriate tool for measuring ecological processes, few empirical studies have attempted to evaluate non-neutrality using these models (e.g. Chust et al. 2013, Hirao et al. 2013, Qiao et al. 2015). Sampling effect is another critical issue that should be considered when disentangling ecological processes; incomplete sampling can create difficulties in identifying the underlying processes of empirical abundance distributions (McGill et al. 2007, White et al. 2012). In this study, we therefore accounted for sampling effects by including species richness and the number of individuals as covariates in the analysis. Nonetheless, tropical forests with large numbers of species (that may generally be equivalent), and temperate or boreal forests with few species produced non-neutral patterns in species abundance distributions, and the regional differences of non-neutrality was significant between biomes. We also detected little effects of sampling size on non-neutrality, which is in line with the previous analysis of Qiao et al. (2015). These findings recall that tree species abundance distributions cannot be regarded simply as a product of random birth, death and speciation. 


\section{Ecological processes of non-neutrality in species abundance distributions}

In contrast to mechanistic non-neutral model used in this study, phenomenological modelling with statistical distributions is one of the fundamental tools often used to evaluate emergent patterns (e.g. log-normal versus log-series or neutral) in species abundance distributions (McGill et al. 2007). Such a phenomenological approach may overlook signals of non-neutrality, because different statistical distributions also provide comparable fits (Volkov et al. 2003, Chave 2004). More importantly, as shown by White et al. (2012), the majority of species abundance distributions follow a log-series that is consistent with neutral patterns (Hubbell 2001). Such a predominance of both log-series and neutrality may convey the idea that non-neutral processes are weak relative to the influence of stochastic processes caused by ecological drift and dispersal. For empirical studies, therefore, researchers should pay attention to the way in which neutral theory fails, rather than emphasize the role of ecological drift and the prevalence of neutrality (Hubbell 2001). A more fruitful reasoning is to consider that neutral and niche perspectives are at opposite ends of a continuum of community assembly rules (Chase and Leibold 2003, Gravel et al. 2006).

Beyond a dichotomous view of neutral versus non-neutral, we found geographical patterns of deviations (abundance evenness/unevenness) from neutrality. Our analysis failed at finding a correlation between deviations from neutrality and climatic factors. In contrast, Qiao et al. (2015) examined the $\delta$ value in tree species abundances in 32 plots on mesic mountainous forests of China and reported that abundance unevenness was positively correlated with latitude and negatively with temperature. They also suggested that precipitation was not a limiting factor for tree species diversity. These contrasting findings may be the result of differences in 
geographical scales examined between the studies. In our global-scale study, the degree of abundance unevenness and its environmental drivers differed among the regions. The greatest $\delta$ value, observed in the Western Palearctic, might suggest the influence of ice-age and anthropogenic disturbances on the elimination of rare species (Svenning and Skov 2005). Correlations of the $\delta$ value with temperature or Quaternary precipitation change in some regions suggest that regional climatic filtering occurs in relation to temperature or drought (Fine and Ree 2006). These results indicate that environmental filtering is not universally increasing from warm tropical to cold boreal forests, but might affect differently tree species assembly between and within biomes. Interestingly, a positive correlation between the $\delta$ value and the quadratic terms of temperature indicates that abundance unevenness frequently occurred in regions experiencing climatically benign conditions, as well as in colder regions. Based on large-scale inventories of tropical forests, Gentry (1988) demonstrated a global-scale pattern of species dominance exists related to family-specific niches, and suggested that species diversity is associated with in situ diversification within particular families. Our findings of non-neutral unevenness in species abundance may reflect an evolutionary imprint of species radiations in tropical climatic conditions (Fine and Kembel 2011). Additionally, abundance evenness caused by negative density-dependent effects was not evident in particular regions in relation to climatic conditions, although it became obvious in areas experiencing greater change in Quaternary precipitation. Hille Ris Lambers et al. (2002) did not find significant differences in negative density-dependent mortality among tropical and temperate forests. Our results also supported the idea that this process may not be prevalent in structuring tree species abundance distributions in tropical biomes. 


\section{Conclusion}

Non-neutral unevenness of tree species abundances was ubiquitous across tropical, temperate, and boreal forests, and was not linearly correlated with macro-scale climatic factors. These findings suggest that large-scale environmental factors drive the appearance of non-neutrality in species abundance distribution, but the processes generating particular dominant species in local communities may be more idiosyncratic; some differences in ecological and/or evolutionary processes exist among or within tropical, temperate, and boreal forest communities (Currie et al. 2004). Also, the regional differences of non-neutrality in tree species abundance among continents may contribute to diversity anomalies related to the region-specific historical processes that are tied to geography and paleoclimate (Gentry 1988, Latham and Ricklefs 1993, Qian and Ricklefs 2000, Fine and Ree 2006). This study illustrated that mechanistic neutral/non-neutral models serve as a helpful tool when ecologists examine the roles of historical and ecological processes in community assemblies.

Acknowledgments - We thank Kana Kubota for assistance with the compilation of the data. We also thank Thorsten Wiegand, James Rosindell, and one anonymous referee for their constructive and insightful comments. Financial support was provided by the Japan Society for the Promotion of Science (nos. 21310025, 21247006).

\section{References}

Alonso, D. et al. 2006. The merits of neutral theory. - Trends Ecol. Evol. 21: 451-457.

Chase, J. M. and Leibold, M. A. 2003. Ecological niches: linking classical and 
contemporary approaches. - Chicago Univ. Press.

Chave, J. 2004. Neutral theory and community ecology. - Ecol. Lett. 7: 241-253.

Chust, G. et al. 2013. Latitudinal phytoplankton distribution and the neutral theory of biodiversity. - Global Ecol. Biogeogr. 22: 531-543.

Clark, J. S. 2008. Beyond neutral science. - Trends Ecol. Evol. 214: 8-15.

Currie, D. J. et al. 2004. Predictions and tests of climate-based hypotheses of broad-scale variation in taxonomic richness. - Ecol. Lett. 7: 1121-1134.

Davies, T. J. et al. 2011. Neutral biodiversity theory can explain the imbalance of phylogenetic trees but not the tempo of their diversification. - Evolution 65: $1841-1850$.

Diniz-Filho, J.A.F. and Bini, L.M. 2005. Modelling geographical patterns in species richness using eigenvector-based spatial filters. - Global Ecol. Biogeogr. 14: $177-185$.

Donoghue, M. J. 2008. A phylogenetic perspective on the distribution of plant diversity. - Proc. Natl Acad. Sci. USA 105: 11549-11555.

Etienne, R. S. 2005. A new sampling formula for neutral biodiversity. - Ecol. Lett. 8: $253-260$.

Etienne, R. S. and Alonso, D. 2005. A dispersal-limited sampling theory for species and alleles. - Ecol. Lett. 8: 1147-1156.

Fine, V. A. F. and Kembel, S. W. 2011. Phylogenetic community structure and phylogenetic turnover across space and edaphic gradients in western Amazonian tree communities. - Ecography 34: 552-565.

Fine, P. V. and Ree, R. H. 2006. Evidence for a time - integrated species - area effect on the latitudinal gradient in tree diversity. - Am. Nat. 168: 796-804. 
Fox, J. and Weisberg, S. 2014. car: Companion to Applied Regression. Available at: http://cran.r-project.org/web/packages/car/index.html

Gentry, A. H. 1988. Changes in plant community diversity and floristic composition on environmental and geographical gradients. - Ann. Missouri Bot. Gard. 75: 1-34.

Gilbert, B and Lechowicz, M. J. 2004. Neutral, niches, and dispersal in a temperate forest understory. - Proc. Natl Acad. Sci. USA 101: 7651-7656.

Gotelli, N. J. and McGill, B. J. 2006. Null versus neutral models: what's the difference? - Ecography 29: 793-800.

Gravel, D. et al. 2006. Reconciling niche and neutrality: the continuum hypothesis. Ecol. Lett. 9: 399-409.

Harms, K. E. et al. 2000. Pervasive density-dependent recruitment enhances seedling diversity in a tropical forest. - Nature 404: 493-495.

Harrison, S. and Cornell, H. 2008. Toward a better understanding of the regional causes of local community richness. - Ecol. Lett. 11: 969-979.

Hijmans, R. J. et al. 2013. Geosphere: spherical trigonometry for geographic applications version 1.2-28. Available at: http://cran.r-project.org/web/packages/geosphere/index.html

Hille Ris Lambers, J. et al. 2002. Density-dependent mortality and the latitudinal gradient in species diversity. - Nature 417: 732-735.

Hirao, T. et al. 2013. Species abundance distributions of moth and beetle assemblages in a cool-temperate deciduous forest. - Insect Conserv. Divers. 6: 494-501.

Hubbell, S. P. 2001. The unified neutral theory of biodiversity and biogeography. Princeton University Press.

Hutchinson, G. E. 1959. Homage to Santa Rosalia or why are there so many kinds of 
animals? - Am. Nat. 93: 145-159.

Jabot, F. 2010. A stochastic dispersal-limited trait-based model of community dynamics. - J. Theor. Biol. 262: 650-661.

Jabot, F. and Chave, J. 2009. Inferring the parameters of the neutral theory of biodiversity using phylogenetic information and implications for tropical forests. - Ecol. Lett. 12: 239-248.

Jabot, F. et al. 2008. Reconciling neutral community models and environmental filtering: theory and an empirical test. - Oikos 117: 1308-1320.

Jabot, F. and Chave, J. 2011. Analyzing tropical forest tree species abundance distributions using a nonneutral model and through Approximate Bayesian inference. - Am. Nat. 178: E37-E47.

Jiménez, I. and Ricklefs, R. E. 2014. Diversity anomalies and spatial climate heterogeneity. - Global Ecol. Biogeogr. 23: 988-999.

Latham, R. E. and Ricklefs, R. E. 1993. Continental comparisons of temperate-zone tree species diversity. - In: Ricklefs R. E.and Schluter D. (ed.), Species diversity in ecological communities: historical and geographical perspectives. - University of Chicago Press, pp. 294-314.

Latimer, A. M. et al. 2005. Neutral ecological theory reveals isolation and rapid speciation in a biodiversity hot spot. - Science 309: 1722-1725.

McGill, B. J. 2010. Towards a unification of unified theories of biodiversity. - Ecol. Lett. 13: 627-642.

McGill, B. J. et al. 2007. Species abundance distributions: moving beyond single prediction theories to integration within an ecological framework. - Ecol. Lett. 10: 995-1015. 
Qian, H. and Ricklefs, R. E. 2000. Large-scale processes and the Asian bias in species diversity of temperate plants. - Nature 407: 180-182.

Qiao, X. et al. 2015. A latitudinal gradient in tree community assembly processes evidenced in Chinese forests. - Global Ecol. Biogeogr. DOI: 10.1111/geb.12278.

R Development Core Team 2012. R: a language and environment for statistical computing. R Foundation for Statistical Computing.

Ricklefs, R. E. 2006. The unified neutral theory of biodiversity: do the numbers add up? - Ecology 87: 1424-1431.

Ricklefs, R. E. and Renner, S. S. 2012. Global correlations in tropical tree species richness and abundance reject neutrality. - Science 335: 464-467.

Rosindell, J. and Cornell, S. J. 2009. Species-area curves, neutral models, and long-distance dispersal. - Ecology 90: 1743-1750.

Rosindell, J. et al. 2012. The case for ecological neutral theory. - Trends Ecol. Evol. 27: 203-208.

Rosindell, J. and Phillimore, A. B. 2011. A unified model of island biogeography sheds light on the zone of radiation. - Ecol. Lett. 14: 552-560.

Serra, F. et al. 2013. Neutral theory predicts the relative abundance and diversity of genetic elements in a broad array of Eukaryotic genomics. - PloS ONE 8: e63915.

ter Steege, H. and Zagt, R. J. 2002. Ecology: Density and diversity. - Nature 417: 698699.

Svenning, J. C. and Skov, F. 2005. The relative roles of environment and history as controls of tree species composition and richness in Europe. - J. Biogeogr. 32: 
1019-1033.

Volkov, I. et al. 2003. Neutral theory and relative species abundance in ecology. Nature 424: 1035-1037.

Volkov, I. et al. 2005. Density dependence explains tree species abundance and diversity in tropical forests. - Nature 438: 658-661.

White, E. P. et al. 2012. Characterizing species abundance distributions across taxa and ecosystems using a simple maximum entropy model. - Ecology 93: 1772-1778.

Wiens, J. J. and Donoghue, M. J. 2004. Historical biogeography, ecology and species richness. - Trends Ecol. Evol. 19: 639-644.

\section{Supplementary material}

Supplementary material may be posted as electronic appendices on the journal's appendix site:

Appendix 1 List of data sources for species abundance data.

Appendix 2 Plot data, including information on the geographical distribution and parameter estimates of the neutral and non-neutral models.

Appendix 3 Distributions of plot area (a), minimum measured DBH (b), species richness (c), and the number of individuals sampled in forest plots (d). Note that the values were $\log$ transformed.

Appendix 4 Species abundance distribution (SAD) for each plot and parameter 
estimates ( $m, \theta$, and $\delta$ ) of the neutral and non-neutral models. The number of individuals $(J)$ and species richness $(S)$ sampled in each plot are also shown. Types of species-abundance data were also shown (A: abundance; D: density; RA: relative abundance; RD: relative density). The parameters of non-neutral model are the number of individuals $(J)$, species richness $(S)$, species immigration probability from a metacommunity $(m)$, fundamental biodiversity number $(\theta)$, and the deviation from a neutral pattern $(\delta)$. Red and blue indicate positive and negative $\delta$ values, respectively.

Appendix 5 Regional differences in the parameters of the non-neutral model $(m, \theta$, and $\delta$ ). The mean values for each region were compared using analysis of variance within the two biogeographical realms (in and outside the Holarctic). Values in parenthesis represent standard error.

Appendix 6 Regression coefficients in a polynomial regression model explaining the global or regional scale patterns in $\delta$ for the all plots $(\mathrm{N}=1217)$. Environmental factors of deviations from neutrality were analyzed by generalized linear regression models in which the $\delta$ value of non-neutrality model was set as a response variable. Linear and quadratic terms of elevation (Elev), temperature (Temp), precipitation (Prec), Quaternary temperature change (Q.tc), and Quaternary precipitation change (Q.pc) were set as explanatory variables. Plot area (Area), minimum measured DBH (Size), species richness $(S R), \log$-scaled number of individuals $(\log . J)$, and the first eigenvector of a geographical distance matrix among the plots $(s p P C 1)$ were also included as covariates to evaluate the effects of plot-data bias among the plots. Identity-link functions were 
used. Statistical significances of the coefficients were tested by Wald tests and represented by bold $(\mathrm{P}<0.05)$.

Author contributions: YK conceived the study idea; YK, BK, and TS collected data; FJ developed the statistical approach; BK and YK conducted the analyses; WU and FJ contributed to interpreting the results. YK wrote the first draft, and all authors contributed substantially to revisions.

\section{Figure legends}

Figure 1. Examples of species abundance distribution in forest plots. (a) Abundance evenness with a negative $\delta$ value in non-neutrality model. (b) Abundance unevenness with a positive $\delta$ value in non-neutrality model. The number of individuals $(J)$, species richness $(S)$ sampled in each plot, and the deviation from a neutral pattern $(\delta)$ are also shown.

Figure 2. Sampling effects on the appearance of non-neutrality in forest plots. (a) Relationship between the number of individuals and $\delta$ values in the non-neutrality model. (b) Relationship between species richness and $\delta$ values in the non-neutrality model. Grey bars in (a) and (b) indicate the 95\% confidence interval of $\delta$ values estimated by the non-neutrality model.

Figure 3. Geographical pattern of non-neutrality in tree species abundance distributions. When $\delta$ is negative, individuals of dominant species face increased mortality rates compared with individuals of rare species, indicating that negative density dependent 
mortality results in abundance evenness. When $\delta$ is positive, individuals of rare species face increased mortality rates compared with those of dominant species, indicating that positive density dependent mortality related to environmental filtering results in abundance unevenness (Jabot and Chave 2011). The total number of plots in each region was: Eastern Palearctic, 124; Indo-Pacific, 334; Nearctic, 95; South America, 378; Australia, 40; Africa, 143; and Western Palearctic, 103.

Figure 4. Non-neutral patterns in geographical regions. The red and blue panels indicate the regions outside and within the Holarctic, respectively. Vertical lines represent the 95\% confidence intervals of the mean values of $\delta$ values for each region. When $\delta$ is negative, individuals of dominant species face increased mortality rates compared with individuals of rare species, indicating that negative density dependent mortality results in abundance evenness. When $\delta$ is positive, individuals of rare species face increased mortality rates compared with those of dominant species, indicating that positive density dependent mortality related to environmental filtering results in abundance unevenness (Jabot and Chave 2011). 
1 Table 1. Regression coefficients in the multiple regression models explaining the global or regional scale patterns in non-neutrality $(\delta)$

2 for the all plots $(\mathrm{N}=1,168)$. Environmental factors of deviations from neutrality were analyzed by generalized linear regression models

3 in which the $\delta$ value of non-neutrality model was set as a response variable. Elevation (Elev), temperature (Temp), precipitation (Prec),

4 Quaternary temperature change $(Q . t c)$ and Quaternary precipitation change $(Q . p c)$ were set as explanatory variables. Plot area (Area),

5 minimum measured DBH (Size), species richness $(S R)$, log-scaled number of individuals ( $\operatorname{Log.J})$, and the first eigenvector of a

6 geographical distance matrix among the plots $(s p P C 1)$ were also included as covariates to evaluate the effects of plot-data bias among

7 the plots. Identity-link function was used for $\delta$. Statistical significances of the coefficients were tested by Wald tests $(* P<0.05$, $* * P<$

$8 \quad 0.01, * * * P<0.001)$.

\begin{tabular}{lllllllllll}
\hline Region & Elev & Temp & Prec & Q.tc & Q.pc & Area & Size & SR & Log.J & spPC1 \\
\hline Global & -0.02 & 0.02 & 0 & -0.01 & $-0.06^{* * *}$ & 0.01 & 0.01 & 0.01 & 0 & 0.02 \\
South America & 0.11 & 0.11 & 0 & 0.05 & -0.01 & 0 & $-0.06^{* *}$ & 0.02 & 0 & -0.05 \\
Africa & -0.07 & $0.15^{*}$ & -0.02 & 0.04 & $-0.16^{*}$ & 0.06 & 0.1 & 0.09 & -0.05 & -0.05 \\
Indo-Pacific & 0.04 & 0.06 & 0.01 & $-0.08^{* *}$ & $-0.1^{* * *}$ & -0.05 & -0.04 & -0.01 & $0.09 *$ & 0.03 \\
Australia & 0.23 & -0.06 & -0.17 & -0.06 & 0.08 & 0.13 & -0.17 & 0.05 & 0.05 & 0.07 \\
Nearctic & 0.03 & 0.22 & -0.07 & 0.22 & 0 & 0.08 & 0.05 & 0.01 & $-0.22^{* * *}$ & 0.03 \\
Western Palearctic & 0.01 & 0.05 & -0.02 & -0.01 & 0.01 & -0.09 & 0.02 & $-0.17^{* *}$ & 0.04 & -0.01 \\
Eastern Palearctic & 0.13 & 0.15 & 0.04 & 0.08 & -0.05 & -0.02 & -0.07 & -0.01 & 0.05 & 0.07 \\
\hline
\end{tabular}


$9 \quad$ Fig.1

(a)

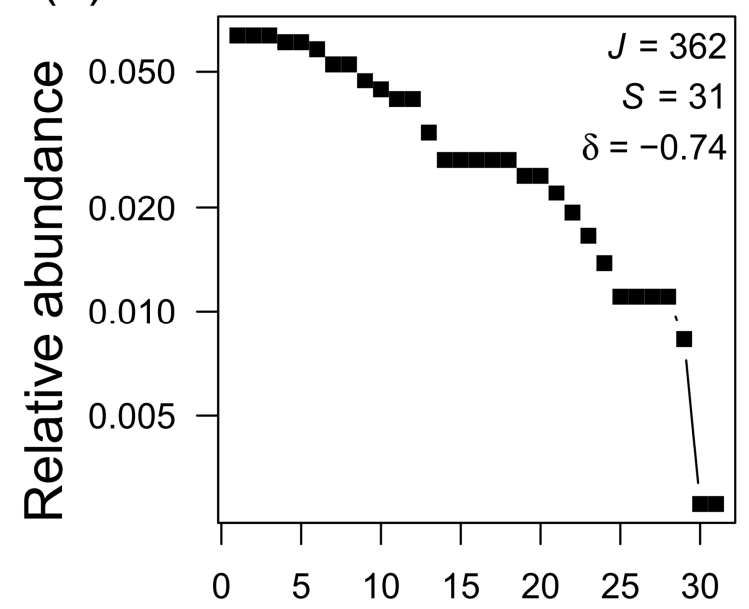

(b)

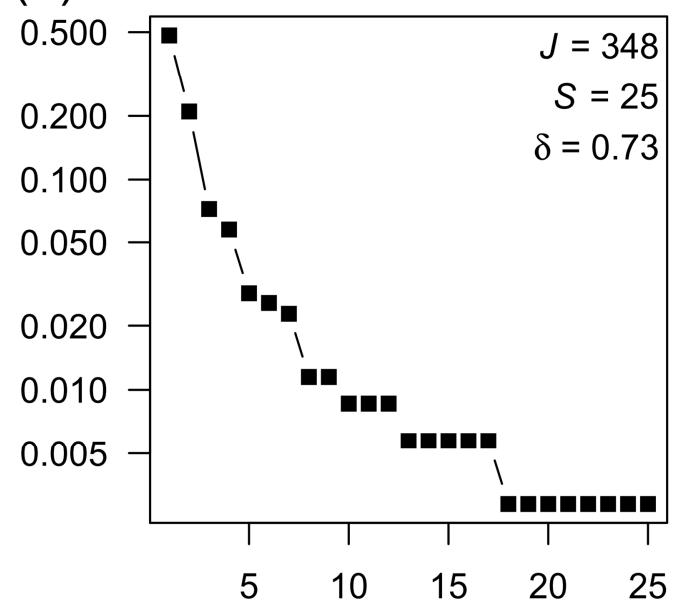


Fig. 2
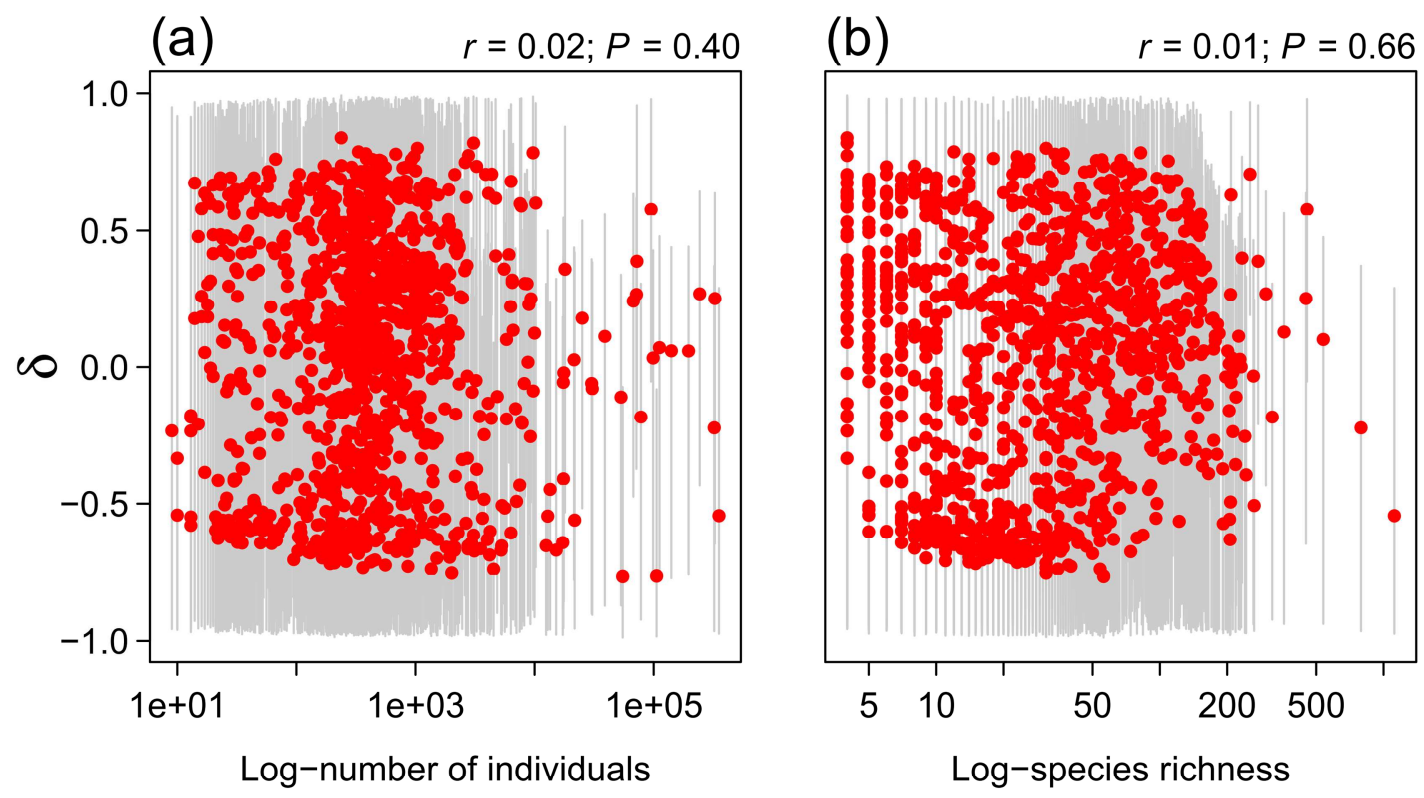
16

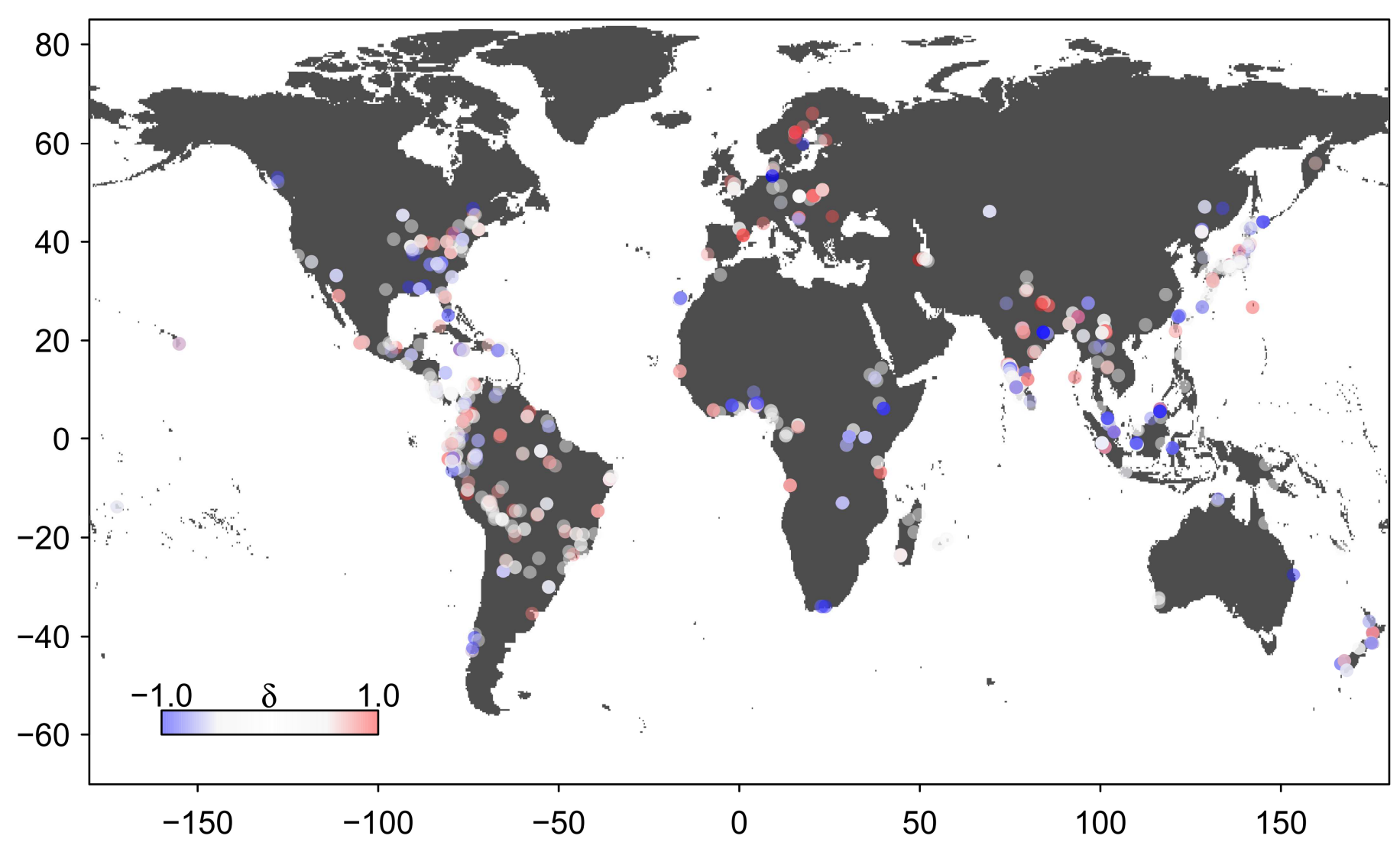


Fig. 4

19

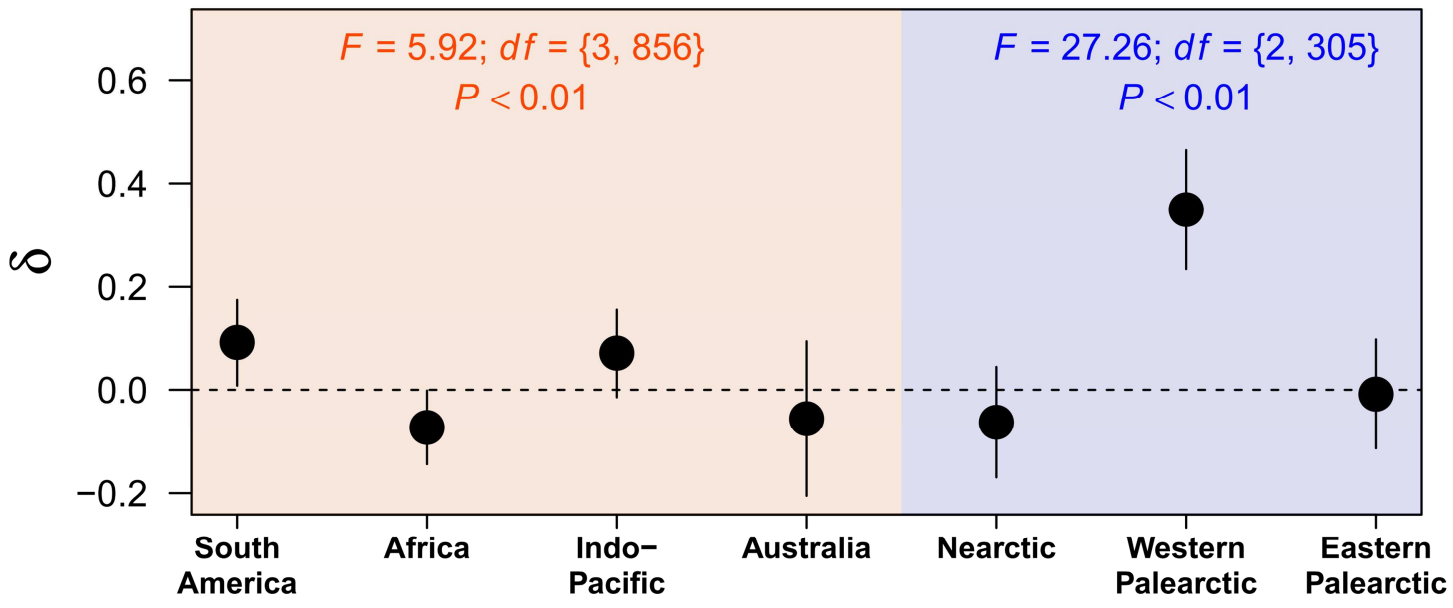

